\title{
Constraining the magnetic field in the EM170817 ejecta with H.E.S.S. observations
}

\author{
Stefan Ohm ${ }^{1}$ \\ Deutsches Elektronen-Synchrotron DESY, Zeuthen, Germany \\ E-mail: stefan.ohmedesy.de
}

for the H.E.S.S. Collaboration ${ }^{2}$

E-mail: contact.hess@hess-experiment.eu

The detection of the first electromagnetic counterpart to the binary neutron star merger remnant GW170817 established the connection between short gamma-ray bursts and binary neutron star mergers as well as it confirmed the forging of heavy elements in the ejecta (a socalled kilonova) via the $r$ process. The brightening of non-thermal radio and X-ray emission, however, came with some surprise and lasted more than 100 days. Current theoretical models attempt to explain the light curve as either originating from a relativistic off-axis jet, or a kilonova-like outflow. Combining the VLA and Chandra measurements with observations in the $\mathrm{GeV}-\mathrm{TeV}$ domain, can help to break the ambiguity between energy in non-thermal electrons and magnetic field of the emission region, almost independent of the assumed origin of the emission. Here we report for the first time on deep H.E.S.S. observations of EM170817 between 120 and 270 days after the binary neutron star merger with the full H.E.S.S. array of telescopes as well as on updated prompt ( $<5$ days) observations with the upgraded HESS phase-I telescopes. Implications of the H.E.S.S. measurement for the magnetic field in the merger remnant are discussed in the context of a isotropic, non-relativistic scenario and in a relativistic, offaxis jet scenario.

\footnotetext{
${ }^{1}$ Speaker

${ }^{2}$ for collaboration list see PoS(ICRC2019)1177

! Copyright owned by the author(s) under the terms of the Creative Commons 